\title{
Analysis of tree algorithm for collision resolution ${ }^{\dagger}$
}

\author{
László Györfi and Sándor Győri
}

Department of Computer Science and Information Theory, Budapest University of Technology and Economics,

H-1117, Magyar tudósok körútja 2., Budapest, Hungary.

For the tree algorithm introduced by Capetanakis (1979) and Tsybakov and Mihailov (1978) let $L_{N}$ denote the expected collision resolution time given the collision multiplicity $N$. If $L(z)$ stands for the Poisson transform of $L_{N}$, then we show that $L_{N}-L(N) \simeq 1.29 \cdot 10^{-4} \cos \left(2 \pi \log _{2} N+0.698\right)$.

Keywords: random access communication, collision resolution time, tree algorithm

\section{Collision Channel with Feedback}

In multi-user communications the problem is how to serve many senders if one common communication channel is given. The classical solution is a kind of multiplexing, i.e., either time-division multiplexing, or frequency-division multiplexing. For partially active senders, always there are a large number of senders, each which has nothing to send most of the time. In this communication situation the multiplexing is inefficient. One such situation, namely the problem of communicating from remote terminals on various islands of Hawaii via a common radio channel to the main central computer, led to the invention by Abramson (1970) of the first formal random-access algorithm, now commonly called pure ALOHA and to the design of a radio linked computer network, called ALOHANET (cf. Abramson (1985)). The performance of the ALOHA protocol is very poor if the channel occupancy increases beyond a certain level, in fact for Poisson arrivals it is unstable.

In this paper we consider the multiple-access collision channel with ternary feedback. An unlimited number of users are allowed to transmit packets of a fixed length whose duration is taken as a time unit and called slot. Stations can begin to transmit packets only at times $n \in\{0,1,2,3, \ldots\}$. A slot is a time interval $[n, n+1)$. The destination for the packet contents is a single common receiver. All users send their packets through a common channel. Senders of different packets cannot interchange information. Thus it is convenient to suppose that there are infinitely many non cooperating users and that the packet arrivals can be modelled as a Poisson process in time with intensity $\lambda$.

When two or more users send a packet in the same time slot, these packets "collide" and the packet information is lost, i.e., the receiver cannot determine the packet contents, and retransmission will be necessary. However, all users, also those who were not transmitting can learn-from the ternary feedback just before time instant $n+1$ - the story of time slot $[n, n+1)$ :

- feedback 0 means an idle slot,

- feedback 1 means successful transmission by a single user,

- feedback of the collision symbol $*$ means that collision happened.

A conflict resolution protocol (or random multiple access algorithm) is a retransmission scheme for the packets in a collision. Such a scheme must insure the eventual successful transmission of all these packets.

${ }^{\dagger}$ This work was sponsored by the Office of Naval Research International Field Office and the Air Force Office of Scientific Research, Air Force Material Command, USAF, under grant number FA8655-05-1-3017. The U.S Government is authorized to reproduce and distribute reprints for Governmental purpose notwithstanding any copyright notation thereon. 
A conflict resolution protocol has two components: the channel-access protocol (CAP) and the collision resolution algorithm (CRA).

The CAP is a distributed algorithm that determines, for each transmitter, when a newly arrived packet at that transmitter is sent for the first time. The simplest CAP, both conceptually and practically, is the free-access protocol in which a transmitter sends a new packet in the first slot following its arrival. The blocked-access protocol is that in which a transmitter sends a new packet in the first slot following the resolution of all collisions that had occurred prior to the arrival of the packet.

The CRA can be defined as an algorithm (distributed in space and time) that organizes the retransmission of the colliding packets in such a way that every packet is eventually transmitted successfully with finite delay and all transmitters become aware of this fact.

The time span from the slot where an initial collision occurs up to and including the slot from which all transmitters recognize that all packets involved in the above initial collision have been successfully received is called collision resolution interval (CRI).

\section{Tree Algorithm for Collision Resolution}

Independently of each other, Capetanakis (1979); Tsybakov and Mihailov (1978) introduced the first CRA, called tree algorithm, which resulted in stable conflict resolution protocol.

Let $N$ denote the number of active transmitters, i.e., the multiplicity of the collision. According to the tree algorithm, all active transmitters send the packets in the next slot. If there was no active transmitter $(N=0)$ then the feedback is 0 and the tree algorithm terminates. If there was exactly one active transmitter $(N=1)$ then the feedback is 1 and the transmission was successful, so, again, the algorithm terminates. Otherwise $N \geq 2$, the feedback is the collision symbol $*$. After this collision, all transmitters involved flip a (non-biased) binary coin. Those flipping 0 retransmit in the next slot, those flipping 1 retransmit in the next slot after the collision (if any) among those flipping 0 has been resolved.

The algorithm can be represented by a binary rooted search tree. Collisions correspond to intermediate nodes, while empty slots and successful slots correspond to terminal nodes.

In order to analyze the tree algorithm, let $X$ denote the number of packets sent in the first slot of the CRI, and let $Y$ be the length (in slots) of the same CRI, i.e., the collision resolution time resolving $X$ conflicts. Introduce the notation

$$
L_{N}=\mathbf{E}\{Y \mid X=N\},
$$

then $L_{N}$ is the conditional expectation of the collision resolution time, given the multiplicity of the conflict $N$.

Hajek (1980) indicated first that $L_{N} / N$ does not converge, Massey (1981) bounded the oscillation of $L_{N} / N$, and then Mathys and Flajolet (1985) showed its asymptotic behavior in an implicit way. Janssen and de Jong (2000) clarified the exact asymptotics of $L_{N} / N$ :

$$
\frac{L_{N}}{N}=\frac{2}{\ln 2}+A \sin \left(2 \pi \log _{2} N+\varphi\right)+O\left(N^{-1}\right),
$$

where

$$
A=3.127 \cdot 10^{-6}, \quad \varphi=0.9826 .
$$

These imply that

$$
2.8853869 \leq \liminf _{N \rightarrow \infty} \frac{L_{N}}{N} \leq \limsup _{N \rightarrow \infty} \frac{L_{N}}{N} \leq 2.8853932 .
$$

Introduce the notation

$$
L(z)=\sum_{N=0}^{\infty} L_{N} \frac{z^{N}}{N !} e^{-z} .
$$

$L(z)$ is called the Poisson transform of the sequence $\left\{L_{N}\right\}$ (cf. Szpankowski (2001)).

The asymptotic behavior of $L_{N}$ is mostly investigated in the literature through its Poisson transform $L(z)$. The question naturally arises how small is the difference between $L_{N}$ and $L(N)$ if $N \rightarrow \infty$. Mathys (1984) proved that $L_{N}-L(N)=O(1)$. Next we extend it showing its oscillation. 


\section{Oscillation of $L_{N}-L(N)$}

\section{Theorem 1.}

$$
L_{N}-L(N) \simeq A \cos \left(2 \pi \log _{2} N+\varphi\right)
$$

where

$$
A=1.29 \cdot 10^{-4}, \quad \varphi=0.698 .
$$

Proof. Based on Gulko and Kaplan (1985); Mathys and Flajolet (1985) the formula for $L_{N}$ can be written in a nonrecursive way: $L_{0}=L_{1}=1$, and for $N \geq 2$,

$$
L_{N}=1+2 \sum_{j=0}^{\infty}\left(2^{j}\left(1-\left(1-2^{-j}\right)^{N}\right)-N\left(1-2^{-j}\right)^{N-1}\right) .
$$

Let us calculate the Poisson transform of $L_{N}$ (cf. Mathys and Flajolet (1985). By (1)

$$
\begin{aligned}
L(z) & =\sum_{N=0}^{\infty} L_{N} \frac{z^{N}}{N !} e^{-z} \\
& =\sum_{N=0}^{\infty} \frac{z^{N}}{N !} e^{-z}+2 \sum_{N=2}^{\infty} \sum_{j=0}^{\infty}\left(2^{j}\left(1-\left(1-2^{-j}\right)^{N}\right)-N\left(1-2^{-j}\right)^{N-1}\right) \frac{z^{N}}{N !} e^{-z} \\
& =1+2 \sum_{j=0}^{\infty}\left(2^{j}\left(1-e^{-2^{-j} z}\right)-z e^{-2^{-j} z}\right) .
\end{aligned}
$$

By (1) and (2),

$$
\begin{aligned}
L_{N}-L(N) & =1+2 \sum_{j=0}^{\infty}\left(2^{j}\left(1-\left(1-2^{-j}\right)^{N}\right)-N\left(1-2^{-j}\right)^{N-1}\right)-1-2 \sum_{j=0}^{\infty}\left(2^{j}\left(1-e^{-2^{-j} N}\right)-N e^{-2^{-j} N}\right) \\
& =2 \sum_{j=0}^{\infty} 2^{j}\left(e^{-2^{-j} N}-\left(1-2^{-j}\right)^{N}\right)+2 \sum_{j=0}^{\infty} N\left(e^{-2^{-j} N}-\left(1-2^{-j}\right)^{N-1}\right) \\
& =2 \sum_{j=0}^{\infty} 2^{j} e^{-2^{-j} N}\left(1-\left(e^{2^{-} j}\left(1-2^{-j}\right)\right)^{N}\right)+2 \sum_{j=0}^{\infty} N\left(e^{-2^{-j}} e^{-2^{-j}(N-1)}-\left(1-2^{-j}\right)^{N-1}\right) \\
& =: 2 A+2 B .
\end{aligned}
$$

For getting lower and upper bounds we use the following inequalities. If $0 \leq x \leq 1$, then

$$
\begin{aligned}
& 1+x+\frac{x^{2}}{2} \leq e^{x} \\
& 1-\frac{x^{2}}{2}-\frac{x^{3}}{2} \leq e^{x}(1-x) \leq 1-\frac{x^{2}}{2}-\frac{x^{2}}{2}+\frac{x^{3}}{2} \\
& 1-x \leq e^{-x} \leq 1-\frac{x^{2}}{2} \\
& 1-x+\frac{x^{2}}{2}
\end{aligned}
$$

and if $a \geq b \geq 0$, then

$$
(a-b) N b^{N-1} \leq a^{N}-b^{N} \leq(a-b) N a^{N-1} .
$$

Lower bound:

$$
\begin{aligned}
A & \geq \sum_{j=0}^{\infty} 2^{j} e^{-2^{-j} N}\left(1-\left(1-\frac{2^{-2 j}}{2}\right)^{N}\right) \\
& \geq \sum_{j=0}^{\infty} 2^{j} e^{-2^{-j} N} \frac{2^{-2 j}}{2} N\left(1-\frac{2^{-2 j}}{2}\right)^{N-1} \\
& \geq \frac{1}{2} \sum_{j=0}^{\infty} 2^{-j} N e^{-2^{-j} N}\left(1-(N-1) \frac{2^{-2 j}}{2}\right) \\
& \geq \frac{1}{2} \sum_{j=0}^{\infty} 2^{-j} N e^{-2^{-j} N}\left(1-N \frac{2^{-2 j}}{2}\right)
\end{aligned}
$$




$$
\begin{aligned}
& =\frac{1}{2} \sum_{j=0}^{\infty} 2^{-j} N e^{-2^{-j} N}-\frac{1}{4} \sum_{j=0}^{\infty} 2^{-3 j} N^{2} e^{-2^{-j} N} \\
& =: I_{1}+I_{2},
\end{aligned}
$$

and

$$
\begin{aligned}
B \geq & \sum_{j=0}^{\infty} N\left(\left(1-2^{-j}\right) e^{-2^{-j}(N-1)}-\left(1-2^{-j}\right)^{N-1}\right) \\
= & \sum_{j=0}^{\infty} N e^{-2^{-j}(N-1)}\left(1-\left(e^{2^{-j}}\left(1-2^{-j}\right)\right)^{N-1}\right)-\sum_{j=0}^{\infty} 2^{-j} N e^{-2^{-j}(N-1)} \\
\geq & \sum_{j=0}^{\infty}(N-1) e^{-2^{-j}(N-1)}\left(1-\left(1-\frac{2^{-2 j}}{2}\right)^{N-1}\right)-\sum_{j=0}^{\infty} 2^{-j} N e^{-2^{-j}(N-1)} \\
\geq & \frac{1}{2} \sum_{j=0}^{\infty} 2^{-2 j}(N-1)^{2} e^{-2^{-j}(N-1)}\left(1-\frac{2^{-2 j}}{2}\right)^{N-1}-\sum_{j=0}^{\infty} 2^{-j} N e^{-2^{-j}(N-1)} \\
\geq & \frac{1}{2} \sum_{j=0}^{\infty} 2^{-2 j}(N-1)^{2} e^{-2^{-j}(N-1)}\left(1-(N-1) \frac{2^{-2 j}}{2}\right)-\sum_{j=0}^{\infty} 2^{-j} N e^{-2^{-j}(N-1)} \\
= & \frac{1}{2} \sum_{j=0}^{\infty} 2^{-2 j}(N-1)^{2} e^{-2^{-j}(N-1)}-\frac{1}{4} \sum_{j=0}^{\infty} 2^{-4 j}(N-1)^{3} e^{-2^{-j}(N-1)} \\
& -\sum_{j=0}^{\infty} 2^{-j}(N-1) e^{-2^{-j}(N-1)}-\sum_{j=0}^{\infty} 2^{-j} e^{-2^{-j}(N-1)} \\
= & : J_{1}+J_{2}+J_{3}+J_{4} .
\end{aligned}
$$

Upper bound:

$$
\begin{aligned}
A & \leq \sum_{j=0}^{\infty} 2^{j} e^{-2^{-j} N}\left(1-\left(1-\frac{2^{-2 j}}{2}-\frac{2^{-3 j}}{2}\right)^{N}\right) \\
& \leq \sum_{j=0}^{\infty} 2^{j} e^{-2^{-j} N} \frac{2^{-2 j}}{2}\left(1+2^{-j}\right) N \\
& =\frac{1}{2} \sum_{j=0}^{\infty} 2^{-j} N e^{-2^{-j} N}\left(1+2^{-j}\right) \\
& =\frac{1}{2} \sum_{j=0}^{\infty} 2^{-j} N e^{-2^{-j} N}+\frac{1}{2} \sum_{j=0}^{\infty} 2^{-2 j} N e^{-2^{-j} N} \\
& =: I_{1}+\widetilde{I}_{2},
\end{aligned}
$$

and

$$
\begin{aligned}
B & \leq \sum_{j=0}^{\infty} N\left(\left(1-2^{-j}+\frac{2^{-2 j}}{2}\right) e^{-2^{-j}(N-1)}-\left(1-2^{-j}\right)^{N-1}\right) \\
& =\sum_{j=0}^{\infty} N\left(e^{-2^{-j}(N-1)}-\left(1-2^{-j}\right)^{N-1}\right)-\sum_{j=0}^{\infty} 2^{-j} N\left(1-\frac{2^{-j}}{2}\right) e^{-2^{-j}(N-1)} \\
& =\sum_{j=0}^{\infty} N e^{-2^{-j}(N-1)}\left(1-\left(e^{2^{-j}}\left(1-2^{-j}\right)\right)^{N-1}\right)-\sum_{j=0}^{\infty} 2^{-j} N\left(1-\frac{2^{-j}}{2}\right) e^{-2^{-j}(N-1)} \\
& \leq \sum_{j=0}^{\infty} N e^{-2^{-j}(N-1)}\left(1-\left(1-\frac{2^{-2 j}}{2}-\frac{2^{-3 j}}{2}\right)^{N-1}\right)-\sum_{j=0}^{\infty} 2^{-j}(N-1)\left(1-\frac{2^{-j}}{2}\right) e^{-2^{-j}(N-1)}
\end{aligned}
$$




$$
\begin{aligned}
\leq & \frac{1}{2} \sum_{j=0}^{\infty} 2^{-2 j} N(N-1)\left(1+2^{-j}\right) e^{-2^{-j}(N-1)}-\sum_{j=0}^{\infty} 2^{-j}(N-1)\left(1-\frac{2^{-j}}{2}\right) e^{-2^{-j}(N-1)} \\
= & \frac{1}{2} \sum_{j=0}^{\infty} 2^{-2 j}(N-1)^{2} e^{-2^{-j}(N-1)}+\sum_{j=0}^{\infty} 2^{-2 j}(N-1) e^{-2^{-j}(N-1)}+\frac{1}{2} \sum_{j=0}^{\infty} 2^{-3 j}(N-1)^{2} e^{-2^{-j}(N-1)} \\
& +\frac{1}{2} \sum_{j=0}^{\infty} 2^{-3 j}(N-1) e^{-2^{-j}(N-1)}-\sum_{j=0}^{\infty} 2^{-j}(N-1) e^{-2^{-j}(N-1)} \\
= & : J_{1}+2 \widetilde{J}_{2}+\widetilde{J}_{3}+\widetilde{J}_{4}+J_{3}
\end{aligned}
$$

It can be shown that if $N \rightarrow \infty$, then $I_{2}, \widetilde{I}_{2}, J_{2}, J_{4}, \widetilde{J}_{2}, \widetilde{J}_{3}, \widetilde{J}_{4}$ all tend to 0 . Both from the upper and the lower bounds the same terms remain, so we have derived the following asymptotical equation which should be analyzed further.

$$
L_{N}-L(N)=2(A+B)=2\left(I_{1}+J_{1}+J_{3}\right)+o(1)
$$

It can be further simplified by showing that $2 I_{1}+J_{3} \rightarrow 0$ if $N \rightarrow \infty$. Let us lower bound it firstly,

$$
\begin{aligned}
2 I_{1}+J_{3} & =\sum_{j=0}^{\infty} 2^{-j} N\left(e^{-2^{-j}}-1\right) e^{-2^{-j}(N-1)}+\sum_{j=0}^{\infty} 2^{-j} e^{-2^{-j}(N-1)} \\
& \geq \sum_{j=0}^{\infty} 2^{-j} N\left(1-2^{-j}-1\right) e^{-2^{-j}(N-1)}+\sum_{j=0}^{\infty} 2^{-j} e^{-2^{-j}(N-1)} \\
& =-\sum_{j=0}^{\infty} 2^{-2 j} N e^{-2^{-j}(N-1)}+\sum_{j=0}^{\infty} 2^{-j} e^{-2^{-j}(N-1)}
\end{aligned}
$$

and then upper bound it

$$
\begin{aligned}
2 I_{1}+J_{3} & \leq \sum_{j=0}^{\infty} 2^{-j} N\left(1-2^{-j}+\frac{2^{-2 j}}{2}-1\right) e^{-2^{-j}(N-1)}+\sum_{j=0}^{\infty} 2^{-j} e^{-2^{-j}(N-1)} \\
& =-\sum_{j=0}^{\infty} 2^{-2 j} N\left(1-\frac{2^{-j}}{2}\right) e^{-2^{-j}(N-1)}+\sum_{j=0}^{\infty} 2^{-j} e^{-2^{-j}(N-1)}
\end{aligned}
$$

Notice that both bounds tend to 0 . That is why $L_{N}-L(N)$ asymptotically equals to

$$
\begin{aligned}
L_{N}-L(N) & =2 J_{1}+J_{3}+o(1) \\
& =\sum_{j=0}^{\infty} 2^{-2 j}(N-1)^{2} e^{-2^{-j}(N-1)}-\sum_{j=0}^{\infty} 2^{-j}(N-1) e^{-2^{-j}(N-1)}+o(1) \\
& =: \Delta(N-1)+o(1)
\end{aligned}
$$

The technique being used here is Mellin transform (cf. Szpankowski (2001)). Reader can find an excellent survey on Mellin transform in Flajolet et al. (1995), and some application of Mellin transform to similar problems in Knuth (1973) pages 131-134, and Jacquet and Regnier (1986). The Mellin transform of a complex valued function $f(x)$ defined over positive reals is

$$
\mathcal{M}[f(x) ; s]=F(s)=\int_{0}^{\infty} x^{s-1} f(x) d x, \quad a<\Re(s)<b,
$$

where $(a, b)$ is the fundamental (convergence) strip and $\Re(\cdot)(\Im(\cdot))$ denotes the real (imaginary) part of its argument. The inversion formula is

$$
f(x)=\frac{1}{2 \pi i} \int_{c-i \infty}^{c+i \infty} x^{-s} F(s) d s, \quad a<c<b,
$$


where $c$ is an arbitrary real number from the fundamental strip $(a, b)$.

One of the basic properties of the Mellin transform is that if

$$
\mathcal{M}[f(x) ; s]=F(s), \quad a<\Re(s)<b,
$$

then

$$
\mathcal{M}\left[\alpha x^{\beta} f(\gamma x) ; s\right]=\alpha \gamma^{-s} F(s+\beta), \quad a-\beta<\Re(s)<b-\beta .
$$

If we consider an elementary Mellin transform (cf. Szpankowski (2001) page 401):

$$
\mathcal{M}\left[e^{-x} ; s\right]=\Gamma(s), \quad 0<\Re(s)<\infty,
$$

then from (3) and (4) we have

$$
\begin{aligned}
\mathcal{M}\left[x^{2} e^{-x} ; s\right] & =\Gamma(s+2), & & -2<\Re(s)<\infty, \\
\mathcal{M}\left[x e^{-x} ; s\right] & =\Gamma(s+1), & & -1<\Re(s)<\infty,
\end{aligned}
$$

where $\Gamma(\cdot)$ denotes the complete gamma function that is in Euler's limit form (cf. Szpankowski (2001) page 41):

$$
\Gamma(s)=\lim _{n \rightarrow \infty} \frac{n^{s} n !}{s(s+1)(s+2) \cdots(s+n)} .
$$

By applying Mellin transform technique the difference $\Delta(N)$ can be expressed in terms of the gamma function. Thus, with using (5) and (6) (while considering property (3)) we have

$$
\begin{aligned}
\mathcal{M}[\Delta(N) ; s] & =\sum_{j=0}^{\infty} \mathcal{M}\left[2^{-2 j} N^{2} e^{-2^{-j} N} ; s\right]-\sum_{j=0}^{\infty} \mathcal{M}\left[2^{-j} N e^{-2^{-j} N} ; s\right] \\
& =\sum_{j=0}^{\infty}\left(2^{-j}\right)^{-s} \Gamma(s+2)-\sum_{j=0}^{\infty}\left(2^{-j}\right)^{-s} \Gamma(s+1) \\
& =\frac{\Gamma(s+2)}{1-2^{s}}-\frac{\Gamma(s+1)}{1-2^{s}},
\end{aligned}
$$

where $-1<\Re(s)<0$ (in the last step $\Re(s)<0$ is needed for the convergence). Let us choose $c:=-1 / 2$. From the inversion formula it follows that

$$
\Delta(N)=\frac{1}{2 \pi i} \int_{c-i \infty}^{c+i \infty} N^{-s}\left(\frac{\Gamma(s+2)}{1-2^{s}}-\frac{\Gamma(s+1)}{1-2^{s}}\right) d s .
$$

This line integral can be evaluated by using Cauchy's residue theorem (cf. Figure 1). For this calculation some residues are needed. $\frac{1}{1-2^{s}}$ has simple poles at the roots of equation $2^{s}=1$, so if $s=\frac{2 k \pi i}{\ln 2}$

$$
\operatorname{res}_{s=s_{0}} \frac{1}{1-2^{s}}=\lim _{s \rightarrow s_{0}} \frac{1}{\left(1-2^{s}\right)^{\prime}}=\lim _{s \rightarrow s_{0}} \frac{1}{-2^{s} \ln 2}=-\frac{1}{\ln 2},
$$

for all $s_{0} \in\left\{\frac{2 k \pi i}{\ln 2}, k \in \mathbb{Z}\right\}$.

If we close the integration contour of the inversion integral in the right half plane (and negate the result because of the negative direction of the integration contour), we get

$$
\begin{aligned}
& \Delta(N)=-\frac{1}{2 \pi i}\left(2 \pi i \sum_{k=-\infty}^{\infty} \underset{s=\frac{2 k \pi i}{\ln 2}}{\operatorname{res}} N^{-s}\left(\frac{\Gamma(s+2)}{1-2^{s}}-\frac{\Gamma(s+1)}{1-2^{s}}\right)\right) . \\
L_{N}-L(N)= & \Delta(N-1)+o(1) \\
\simeq & \frac{1}{\ln 2}+\frac{1}{\ln 2} \sum_{k \neq 0} \Gamma\left(2+\frac{2 k \pi i}{\ln 2}\right) e^{-2 k \pi i \log _{2}(N-1)}-\left(\frac{1}{\ln 2}+\frac{1}{\ln 2} \sum_{k \neq 0} \Gamma\left(1+\frac{2 k \pi i}{\ln 2}\right) e^{-2 k \pi i \log _{2}(N-1)}\right) \\
= & \frac{1}{\ln 2} \sum_{k \neq 0}\left(\Gamma\left(2+\frac{2 k \pi i}{\ln 2}\right)-\Gamma\left(1+\frac{2 k \pi i}{\ln 2}\right)\right) e^{-2 k \pi i \log _{2}(N-1)}
\end{aligned}
$$




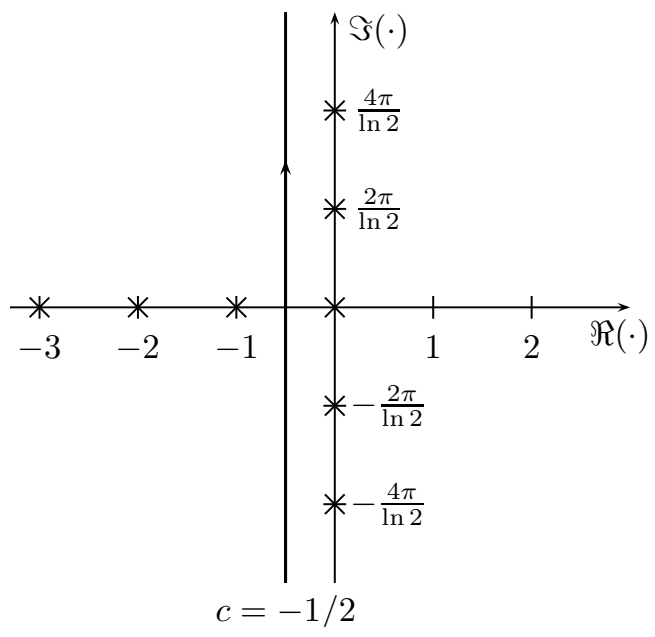

Fig. 1: Poles of $\mathcal{M}[\Delta(N) ; s]$ and the line integral for the inversion formula

As the gamma function decays exponentially fast over imaginary lines, for $L_{N}-L(N)$ a sharp approximation can be given by (8) if we take into account just the first two terms (for $k= \pm 1$ ) of the sum, i.e., the approximation error is of order $10^{-9}$.

$$
L_{N}-L(N) \simeq A \cos \left(2 \pi \log _{2}(N-1)+\varphi\right),
$$

where

$A=\frac{2}{\ln 2}\left(\left(\Re\left(\Gamma\left(2+\frac{2 \pi i}{\ln 2}\right)-\Gamma\left(1+\frac{2 \pi i}{\ln 2}\right)\right)\right)^{2}+\left(\Im\left(\Gamma\left(2+\frac{2 \pi i}{\ln 2}\right)-\Gamma\left(1+\frac{2 \pi i}{\ln 2}\right)\right)\right)^{2}\right)^{1 / 2}=1.29 \cdot 10^{-4}$,

and

$\varphi=\operatorname{arctg} \frac{\Im\left(\Gamma\left(2+\frac{2 \pi i}{\ln 2}\right)-\Gamma\left(1+\frac{2 \pi i}{\ln 2}\right)\right)}{\Re\left(\Gamma\left(2+\frac{2 \pi i}{\ln 2}\right)-\Gamma\left(1+\frac{2 \pi i}{\ln 2}\right)\right)}=0.698$.

Thus

$$
\left|L_{N}-L(N)\right| \lesssim A=1.29 \cdot 10^{-4} .
$$

In order to finish the proof we have to show that if $N \rightarrow \infty$

$$
\cos \left(2 \pi \log _{2} N+\varphi\right)-\cos \left(2 \pi \log _{2}(N-1)+\varphi\right)=o(1) .
$$

It is easy since

$$
\begin{aligned}
\cos \left(2 \pi \log _{2} N+\varphi\right)-\cos \left(2 \pi \log _{2}(N-1)+\varphi\right)= & \left(\cos \left(2 \pi \log _{2} N\right)-\cos \left(2 \pi \log _{2}(N-1)\right)\right) \cos \varphi(9) \\
& -\left(\sin \left(2 \pi \log _{2} N\right)-\sin \left(2 \pi \log _{2}(N-1)\right)\right) \sin (\varphi 0)
\end{aligned}
$$

Then the first term of (9) can be written as

$$
\begin{aligned}
\cos \left(2 \pi \log _{2} N\right)-\cos \left(2 \pi \log _{2}(N-1)\right) & =-2 \sin \left(\pi\left(\log _{2} N+\log _{2}(N-1)\right)\right) \sin \left(\pi\left(\log _{2} N-\log _{2}(N-1)\right)\right) \\
& =-2 \sin \left(\pi \log _{2}(N(N-1))\right) \sin \left(\pi \log _{2}\left(1+\frac{1}{N-1}\right)\right) .
\end{aligned}
$$

As the function $\log _{2}(\cdot)$ is continuous in 1 and function $\sin (\cdot)$ is continuous in 0

$$
\lim _{N \rightarrow \infty} \sin \left(\pi \log _{2}\left(1+\frac{1}{N-1}\right)\right)=0,
$$

that is why (9) is $o(1)$. With similar reasoning it can be easily seen that (10) is also $o(1)$. So, we have proved that

$$
L_{N}-L(N) \simeq A \cos \left(2 \pi \log _{2} N+\varphi\right)
$$




\section{References}

N. Abramson. The aloha system - another alternative for computer communication. In Proc. 1970 Fall Joint Computer Conference, pages 281-285. AFIPS Press, 1970.

N. Abramson. Development of the alohanet. IEEE Trans. on Information Theory, IT-31:119-123, 1985.

J. I. Capetanakis. Tree algorithms for packet broadcast channels. IEEE Trans. on Information Theory, IT-25:505-515, 1979.

P. Flajolet, X. Gourdon, and P. Dumas. Mellin Transforms and Asymptotics: Harmonic sums. Theoretical Computer Science, 144:3-58, 1995.

E. Gulko and M. Kaplan. Analytic properties of multiple-access trees. IEEE Transactions on Information Theory, 31(2):255-263, 1985.

B. Hajek. Expected number of slots needed for the Capetanakis collision-resolution algorithm. unpublished manuscript, Coordinated Sci. Lab., Univ. of Illinois, Urbana IL, 1980.

P. Jacquet and M. Regnier. Trie partitioning process: limiting distributions. In Proceedings of the 11th colloquium on trees in algebra and programming, pages 196-210, New York, 1986. Springer-Verlag.

A. J. E. M. Janssen and M. J. M. de Jong. Analysis of contention tree algorithms. IEEE Transactions on Information Theory, 46:2163-2172, 2000.

D. E. Knuth. The Art of Computer Programming, volume 3. Addison-Wesley, Reading MA, 1973.

J. L. Massey. Collision-resolution algorithms and random-access communications. In G. Longo, editor, Multi-User Communications Systems, pages 73-137. Springer, 1981.

P. Mathys. Analysis of random-access algorithms. PhD dissertation, Dept. of Elec. Engr., Eidgenössische Technische Hochschule, Zürich, 1984.

P. Mathys and P. Flajolet. $Q$-ary collision resolution algorithms in random-access systems with free or blocked channel access. IEEE Transactions on Information Theory, IT-31(2):217-243, 1985.

W. Szpankowski. Average Case Analysis of Algorithms on Sequences. Wiley, New York, 2001.

B. S. Tsybakov and V. A. Mihailov. Slotted multiaccess packet-broadcasting feedback channel. Problemy Peredachi Informatsii, 14:32-59, 1978. 